\title{
Flux Sliding-mode Observer Design for Sensorless Control of Dual Three-phase Interior Permanent Magnet Synchronous Motor
}

\author{
Jian-Qing Shen*, Lei Yuan ${ }^{\dagger}$, Ming-Liang Chen* and Zhen Xie*
}

\begin{abstract}
A novel equivalent flux sliding-mode observer (SMO) is proposed for dual three-phase interior permanent magnet synchronous motor (DT-IPMSM) drive system in this paper. The DTIPMSM has two sets of Y-connected stator three-phase windings spatially shifted by 30 electrical degrees. In this method, the sensorless drive system employs a flux SMO with soft phase-locked loop method for rotor speed and position estimation, not only are low-pass filter and phase compensation module eliminated, but also estimation accuracy is improved. Meanwhile, to get the regulator parameters of current control, the inner current loop is realized using a decoupling and diagonal internal model control algorithm. Experiment results of 2MW-level DT-IPMSM drives system show that the proposed method has good dynamic and static performances.
\end{abstract}

Keywords: Dual three-phase IPMSM, Equivalent flux linkage, Sliding-mode observer; Sensorless control

\section{Introduction}

Multi-phase motor drive system has been widely used in many applications, especially for high-power applications for their advantages compared to the standard three-phase realizations, such as lower torque pulsations, less dc-link current harmonics, reduced rotor harmonic currents, higher power per ampere ration for the same machine volume, etc. [1-4]. Among different multiphase motor drive solutions, one of the most widely discussed is the VSI fed dual threephase induction machine, having two sets of windings spatially shifted by 30 electrical degrees with isolated neutral points, and there are many literatures for induction, but relatively few research for multi-phase permanent magnet synchronous motor (PMSM), especially for highpower PMSM.

In many high performance variable- speed $\mathrm{AC}$ motor drives, field- oriented or vector control is utilized, and rotor-position mechanical sensor is typically required in this method. However, the presence of mechanical sensor presents several drawbacks, such as increases the cost and size of motors, reduces system reliability, etc. Sensorless control technology can achieve the rotor position and speed estimation through exploiting the electrical information about the motor winding, and using a certain control algorithm, which represents the development direction of the AC motor drive system.

So far, several algorithms have been suggested in the recent literatures to estimate the rotor position and the

$\dagger$ Corresponding Author: National Key Laboratory of Vessel Integrated Power System Technology, Naval University of Engineering, China. (lei.yuan.v@qq.com)

* National Key Laboratory of Vessel Integrated Power System Technology, Naval University of Engineering, China. (hgshen258 @sina.com,ccmmllcc@tom.com, xiezhen171@163.com)

Received: March 20, 2013; Accepted: May 7, 2014 speed of the motor. In the flux estimation methods [5], the rotor flux is estimated by using the integral of the difference between the phase voltage and stator resistance voltage, but these methods are sensitive to machine parameter changes, especially the phased resistance. Drift and saturation problems may cause the controller to lose its synchronization ability consequently, especially at low speed. To get better performance, several improvement schemes to flux estimation have been undertaken in [6-9]. However, some methods designed for surface PMSM are not used for IPMSM, due to the differences between stator inductance on $d$-axis and $q$-axis.

Sliding-mode observer (SMO) is an attractive solution compared with other algorithms due to several benefits, such as high state- estimation accuracy, excellent dynamic properties, robustness to parameter variations, and the ability to handle nonlinear system like the IPMSM very well [10-13]. In this paper, a novel equivalent flux estimation based on SMO technology is proposed for DTIPMSM in a stationary reference frame, which also can be applied to both three-phase surface PMSM and IPMSM. To simplify the structure of the controller, an equivalent flux linkage concept is employed in this method, and the conventional switch sign function is replaced by the sigmoid function to reduce system chattering; Moreover, a minimum four current regulators are presented to obtain better control performance. Simulation and experimental results will be presented to demonstrate the feasibility of the proposed control method.

\section{Mathematical Mode of DT-IPMSM}

Using the vector space decomposition technique [14], 
the machine model can be decoupled into three orthogonal subspaces, which are denoted as $(\alpha, \beta),\left(z_{1}, z_{2}\right)$ and $\left(o_{1}, o_{2}\right)$. For machines with distributed windings, only $(\alpha, \beta)$ components contributed to the useful electromechanical energy conversion, whereas $\left(z_{1}, z_{2}\right)$ and $\left(o_{1}, o_{2}\right)$ components only produce losses. An amplitude invariant decoupling transformation is used as

$$
T_{s}=\frac{1}{3}\left[\begin{array}{cccccc}
1 & -\frac{1}{2} & -\frac{1}{2} & \frac{\sqrt{3}}{2} & -\frac{\sqrt{3}}{2} & 0 \\
0 & \frac{\sqrt{3}}{2} & -\frac{\sqrt{3}}{2} & \frac{1}{2} & \frac{1}{2} & 1 \\
1 & -\frac{1}{2} & -\frac{1}{2} & -\frac{\sqrt{3}}{2} & \frac{\sqrt{3}}{2} & 0 \\
0 & -\frac{\sqrt{3}}{2} & \frac{\sqrt{3}}{2} & \frac{1}{2} & \frac{1}{2} & -1
\end{array}\right]
$$

Transformation (1) is the Clark's matrix for DT-IPMSM motor, $\left(o_{1}, o_{2}\right)$ components are omitted from the consideration since the machine has two isolated neutral points. A rotational transformation is applied next to transform the $(\alpha, \beta)$ components into a synchronously rotating reference frame $(d, q)$, which is suitable for field oriented vector control, i.e.,

$$
T_{r}=\left[\begin{array}{cccc}
\cos \theta_{e} & \sin \theta_{e} & & \\
-\sin \theta_{e} & \cos \theta_{e} & & \\
& & 1 & \\
& & & 1
\end{array}\right]
$$

The circuit equation of DT-IPMSM on the $d-q$ rotating coordinate and $\left(z_{1}, z_{2}\right)$ coordinate are given respectively by

$$
\begin{gathered}
{\left[\begin{array}{l}
u_{d} \\
u_{q}
\end{array}\right]=\left[\begin{array}{cc}
R+p L_{d} & -\omega_{e} L_{q} \\
\omega_{e} L_{d} & R+p L_{q}
\end{array}\right]\left[\begin{array}{l}
i_{d} \\
i_{q}
\end{array}\right]+\left[\begin{array}{c}
0 \\
\omega_{e} \psi_{f}
\end{array}\right]} \\
{\left[\begin{array}{l}
u_{z 1} \\
u_{z 2}
\end{array}\right]=\left[\begin{array}{cc}
R+p L_{z} & 0 \\
0 & R+p L_{z}
\end{array}\right]\left[\begin{array}{l}
i_{z 1} \\
i_{z 2}
\end{array}\right]}
\end{gathered}
$$

where,

$\left[\begin{array}{ll}u_{d} & u_{q}\end{array}\right]^{T}$ voltage on the $d^{-} q$ rotating frame;

$\left[\begin{array}{ll}i_{d} & i_{q}\end{array}\right]^{T}$ current on the $d^{-} q$ rotating frame;

$\left[\begin{array}{ll}i_{z 1} & i_{z 2}\end{array}\right]^{T}$ current on the $z_{1}-z_{2}$ frame;

$\left[\begin{array}{ll}L_{d} & L_{q}\end{array}\right]^{T}$ stator inductance on the $d^{-} q$ rotating frame;

$L_{z}$ stator leakage inductance;

$R$ stator resistance;

$p=d / d t$ differential operator

$\omega_{e}$ angular velocity at electrical angle;

$\psi_{f}$ PM flux linkage
Transforming (3) into stationary reference frame $\alpha-\beta$ axis, (5) is derived

$$
\left[\begin{array}{l}
u_{\alpha} \\
u_{\beta}
\end{array}\right]=\left[\begin{array}{cc}
R+p L_{\alpha} & p L_{\alpha \beta} \\
p L_{\alpha \beta} & R+p L_{\alpha}
\end{array}\right]\left[\begin{array}{l}
i_{\alpha} \\
i_{\beta}
\end{array}\right]+\omega_{e} \psi_{f}\left[\begin{array}{c}
-\sin \theta_{e} \\
\cos \theta_{e}
\end{array}\right]
$$

where, $\left[\begin{array}{ll}u_{\alpha} & u_{\beta}\end{array}\right]^{T}$ is the stator voltage on the $\alpha-\beta$ axes, $\left[\begin{array}{ll}i_{\alpha} & i_{\beta}\end{array}\right]^{T}$ is the stator current on the $\alpha-\beta$ axes, and with

$$
\begin{aligned}
& L_{\alpha}=L_{0}+L_{1} \cos 2 \theta_{e}, L_{\beta}=L_{0}-L_{1} \cos 2 \theta_{e} \\
& L_{\alpha \beta}=L_{1} \sin 2 \theta_{e}, L_{0}=\left(L_{d}+L_{q}\right) / 2, L_{1}=\left(L_{d}-L_{q}\right) / 2 .
\end{aligned}
$$

Eq. (5) contains $2 \theta_{e}$ term, which is not easy for mathematical process. To eliminate the $2 \theta_{e}$ term, term, the impedance matrix is rewritten symmetrically like

$$
\left[\begin{array}{l}
u_{d} \\
u_{q}
\end{array}\right]=\left[\begin{array}{cc}
R+p L_{d} & -\omega_{e} L_{q} \\
\omega_{e} L_{q} & R+p L_{d}
\end{array}\right]\left[\begin{array}{l}
i_{d} \\
i_{q}
\end{array}\right]+\left[\begin{array}{c}
0 \\
\omega_{e} \psi_{m}
\end{array}\right]
$$

where, $\psi_{m}=\left(L_{d}-L_{q}\right)\left(i_{d}-i_{q} / \omega_{e}\right)+\psi_{f}$ is defined as equivalent flux linkage.

The circuit equation on the on the $\alpha-\beta$ axis can be derived as (7)

$$
\left[\begin{array}{l}
u_{\alpha} \\
u_{\beta}
\end{array}\right]=\left[\begin{array}{cc}
R+p L_{d} & \omega_{e}\left(L_{d}-L_{q}\right) \\
-\omega_{e}\left(L_{d}-L_{q}\right) & R+p L_{q}
\end{array}\right]\left[\begin{array}{l}
i_{\alpha} \\
i_{\beta}
\end{array}\right]+\omega_{e} \psi_{m}\left[\begin{array}{c}
-\sin \theta_{e} \\
\cos \theta_{e}
\end{array}\right]
$$

where, $\theta_{e}$ is the rotor position in electrical radians, the PM flux linkage $\psi_{\alpha, \beta}$ projected onto the $\alpha-\beta$ axis can be represented as

$$
\psi=\left[\begin{array}{l}
\psi_{\alpha} \\
\psi_{\beta}
\end{array}\right]=\psi_{m}\left[\begin{array}{c}
\cos \theta_{e} \\
\sin \theta_{e}
\end{array}\right]
$$

From the new model (7)-(8), the DT-IPMSM can be described by a linear state equation as (9). Here, the state variables are stator current $i$ and PM flux linkage $\psi$. Assuming that the electrical system's time constant is smaller enough than the mechanical one, i.e., $\dot{\omega}_{e}=0$, the velocity $\omega_{e}$ is regarded as a constant parameter.

$$
\frac{d}{d t}\left[\begin{array}{c}
i \\
\psi
\end{array}\right]=\left[\begin{array}{cc}
A_{11} & A_{12} \\
0 & A_{22}
\end{array}\right]\left[\begin{array}{c}
i \\
\psi
\end{array}\right]+\left[\begin{array}{c}
B_{1} \\
0
\end{array}\right] u+\left[\begin{array}{c}
0 \\
W_{2}
\end{array}\right]
$$

where, $i=\left[\begin{array}{ll}i_{\alpha} & i_{\beta}\end{array}\right]^{T}, \psi=\left[\begin{array}{ll}\psi_{\alpha} & \psi_{\beta}\end{array}\right]^{T}$.

$$
\begin{aligned}
& A_{11}=-\left(R / L_{d}\right) \cdot I+\omega_{e}\left(1-L_{q} / L_{d}\right) \cdot J, \\
& A_{12}=-\left(\omega_{e} / L_{d}\right) \cdot J, A_{22}=\omega_{e} \cdot J,
\end{aligned}
$$




$$
\begin{aligned}
& B_{1}=\left(1 / L_{d}\right) I, I=\left[\begin{array}{cc}
0 & 1 \\
1 & 0
\end{array}\right], J=\left[\begin{array}{cc}
0 & -1 \\
1 & 0
\end{array}\right], \\
& W_{2}=\left(L_{d}-L_{q}\right)\left(\dot{i}_{d}-\ddot{i}_{q} / \omega_{e}\right)\left[\begin{array}{l}
\cos \theta_{e} \\
\sin \theta_{e}
\end{array}\right]
\end{aligned}
$$

The $W_{2}$ term in (9) is linearization error, this term appears only when $i_{d}$ or $i_{q}$ is changing. However, under the velocity control this happens in a very short time because of the high response of the current control loop. Besides, the proposed SMO has an embedded low-pass filter which can cut off the effect of $W_{2}$.

\section{Flux Sliding-mode Observer Design}

\subsection{Design of the observer}

To achieve the flux linkage $\psi$, the proposed observer as (10) is designed based on the stator current model (9).

$$
\frac{d}{d t}\left[\begin{array}{c}
\hat{i} \\
\hat{\psi}
\end{array}\right]=\left[\begin{array}{cc}
A_{11} & A_{12} \\
0 & A_{22}
\end{array}\right]\left[\begin{array}{c}
\hat{i} \\
\hat{\psi}
\end{array}\right]+\left[\begin{array}{c}
B_{1} \\
0
\end{array}\right] u-K \operatorname{sgn}(\hat{i}-i)
$$

where, "^" denotes the estimated quantities, $\operatorname{sgn}(\cdot)$ is the sign function, $K$ is the designed parameter, and the (10) is the conventional SMO. To reduce the chattering phenomenon, the sign function is replaced by a continuous function, i.e., the sigmoid function, which is defined as

$$
\operatorname{sig}(s)=\left(\frac{2}{1+\exp (-a s)}\right)-1
$$

Here, $a$ is a positive constant that can be adjusted the slope of the sigmoid function. And then, the SMO can be rewritten as

$$
\frac{d}{d t}\left[\begin{array}{c}
\hat{i} \\
\hat{\psi}
\end{array}\right]=\left[\begin{array}{cc}
A_{11} & A_{12} \\
0 & A_{22}
\end{array}\right]\left[\begin{array}{l}
\hat{i} \\
\hat{\psi}
\end{array}\right]+\left[\begin{array}{c}
B_{1} \\
0
\end{array}\right] u-K \operatorname{sig}(\hat{i}-i)
$$

Assuming that the motor parameters $R, L_{d}$ and $L_{q}$ exist parameter errors, defined as follows

$$
\Delta R=\hat{R}-R, \Delta L_{d}=\hat{L}_{d}-L_{d}, \Delta L_{q}=\hat{L}_{q}-L_{q}
$$

where, “^" denotes the estimated quantities, $\Delta R, \Delta L_{d}$ and $\Delta L_{q}$ are the stator resistance error, the $d-q$ inductance errors, respectively.

Considering the parameter variations of the motor, the observer (12) can be equivalent to

$$
\frac{d}{d t}\left[\begin{array}{c}
\hat{i} \\
\hat{\psi}
\end{array}\right]=\left[\begin{array}{cc}
A_{11} & A_{12} \\
0 & A_{22}
\end{array}\right]\left[\begin{array}{l}
\hat{i} \\
\hat{\psi}
\end{array}\right]+\left[\begin{array}{c}
B_{1} \\
0
\end{array}\right] u+\left[\begin{array}{c}
W_{1} \\
0
\end{array}\right]-K \mathrm{~s} i \mathrm{~g}(\hat{i}-i)
$$

where, $W_{1}$ is the parameter error input matrix, defined as follows

$$
W_{1}=\left[\begin{array}{l}
W_{1 \alpha} \\
W_{1 \beta}
\end{array}\right]=\left[\begin{array}{ll}
\Delta A_{11} & \Delta A_{12}
\end{array}\right]\left[\begin{array}{c}
\hat{i} \\
\hat{\psi}
\end{array}\right]+\left[\begin{array}{c}
\Delta B_{1} \\
0
\end{array}\right] u
$$

With

$$
\begin{aligned}
& \Delta A_{11}=\hat{A}_{11}-A_{11}=-\frac{\Delta R L_{d}-\Delta L_{d} R}{\hat{L}_{d} L_{d}} I-\omega_{e} \frac{\Delta L_{q} L_{d}-\Delta L_{d} L_{q}}{\hat{L}_{d} L_{d}} J, \\
& \Delta A_{12}=\hat{A}_{12}-A_{12}=\omega_{e} \frac{\Delta L_{d}}{\hat{L}_{d} L_{d}} J, \Delta B_{1}=\hat{B}_{1}-B_{1}=-\frac{\Delta L_{d}}{\hat{L}_{d} L_{d}} .
\end{aligned}
$$

and $K$ is the designed constant parameter, satisfies

$$
K=\left[\begin{array}{l}
K_{1} \\
K_{2}
\end{array}\right]=\left[\begin{array}{cc}
k_{1}+k^{\prime} & 0 \\
0 & k_{1}+k^{\prime} \\
-L_{d} \cdot\left(k_{1}+k^{\prime}\right) & -\frac{L_{d}}{\omega_{e}} \cdot\left(k_{1}+k^{\prime}\right) \cdot k_{2} \\
\frac{L_{d}}{\omega_{e}} \cdot\left(k_{1}+k^{\prime}\right) \cdot k_{2} & -L_{d} \cdot\left(k_{1}+k^{\prime}\right)
\end{array}\right]
$$

The sliding hyper-plane is defined upon the stator current, i.e., $s=\left[\begin{array}{ll}s_{\alpha} & s_{\beta}\end{array}\right]^{T}=\hat{i}-i=0$. So the stator current estimation error dynamic function can be obtained from (9) and (14) as follows

$$
\frac{d}{d t} s=A_{11} s+A_{12}\left[\begin{array}{c}
\tilde{\psi}_{\alpha} \\
\tilde{\psi}_{\alpha}
\end{array}\right]+W_{1}-K_{1} \operatorname{sig}(s)
$$

where, ' $\sim$ ' denotes the estimated error, such as $\tilde{i}=\hat{i}-i$.

\subsection{Lyapunov stability analysis}

In order to prove the stability of the designed observer, the following Lyapunov function candidate is considered.

$$
V=0.5 s^{2}=0.5\left(\tilde{i}_{\alpha}^{2}+\tilde{i}_{\beta}^{2}\right) .
$$

Differentiating (18) with respect to time and substituting (17) into it, then the following equation is obtained

$$
\begin{aligned}
\dot{V} & =\tilde{i}_{\alpha} \dot{\tilde{i}}_{\alpha}+\tilde{i}_{\beta} \dot{\tilde{i}}_{\beta} \\
& =-\frac{R}{L_{d}} \tilde{i}_{\alpha}^{2}+\frac{1}{L_{d}} \omega_{e} \tilde{\psi}_{\beta} \tilde{i}_{\alpha}+W_{1 \alpha} \tilde{i}_{\alpha}-\left(k_{1}+k^{\prime}\right) \operatorname{sig}\left(\tilde{i}_{\alpha}\right) \tilde{i}_{\alpha} \\
& -\frac{R}{L_{d}} \tilde{i}_{\beta}^{2}-\frac{1}{L_{d}} \omega_{e} \tilde{\psi}_{\beta} \tilde{i}_{\beta}+W_{1 \beta} \tilde{i}_{\beta}-\left(k_{1}+k^{\prime}\right) \operatorname{sig}\left(\tilde{i}_{\beta}\right) \tilde{i}_{\beta} .
\end{aligned}
$$

Assuming that $\max \left(\left\|W_{1 \alpha}\right\|,\left\|W_{1 \beta}\right\|\right)<k^{\prime}$, the equation (19) can be given as 


$$
\begin{gathered}
\dot{V}<-\frac{R}{L_{d}} \tilde{i}_{\alpha}^{2}+\frac{1}{L_{d}} \omega_{e} \tilde{\psi}_{\beta} \tilde{i}_{\alpha}-k_{1} \operatorname{sig}\left(\tilde{i}_{\alpha}\right) \tilde{i}_{\alpha}- \\
\frac{R}{L_{d}} \tilde{i}_{\beta}^{2}-\frac{1}{L_{d}} \omega_{e} \tilde{\psi}_{\beta} \tilde{i}_{\beta}-k_{1} \operatorname{sig}\left(\tilde{i}_{\beta}\right) \tilde{i}_{\beta}
\end{gathered}
$$

According to the Lyapunov stability theory, (20) must be obeyed to guarantee that the observer is stable, i.e., $\dot{V}<0$, the parameter $k_{1}$ can be chosen as

$$
\begin{gathered}
k_{1}>\max \left\{-\frac{R}{L_{d}}\left\|\tilde{i}_{\alpha}\right\|+\frac{1}{L_{d}} \omega_{e} \tilde{\psi}_{\beta} \operatorname{sig}\left(\tilde{i}_{\alpha}\right),\right. \\
\left.-\frac{R}{L_{d}}\left\|\tilde{i}_{\beta}\right\|-\frac{1}{L_{d}} \omega_{e} \tilde{\psi}_{\alpha} \operatorname{sig}\left(\tilde{i}_{\beta}\right)\right\} .
\end{gathered}
$$

Hence, $V$ decays to zero, then $\tilde{i}_{\alpha}$ and $\tilde{i}_{\beta}$ are equal to zero. After sliding-mode motion occurs, i.e., $s=\dot{s}=0$, the followings equation can be obtained from (17).

$$
\left[\begin{array}{c}
\tilde{\psi}_{\alpha} \\
\tilde{\psi}_{\beta}
\end{array}\right]=\left(A_{12}^{-1} K_{1}\right) \mathrm{s} i \mathrm{~g}(s)
$$

The flux linkage estimation error dynamic function can be obtained from (9) and (14) as follows

$$
\frac{d}{d t}\left[\begin{array}{c}
\tilde{\psi}_{\alpha} \\
\tilde{\psi}_{\beta}
\end{array}\right]=A_{22}\left[\begin{array}{c}
\tilde{\psi}_{\alpha} \\
\tilde{\psi}_{\beta}
\end{array}\right]-K_{2} \operatorname{sig}(s)
$$

Substituting (22) into (23), then the following equation is obtained

$$
\frac{d}{d t}\left[\begin{array}{c}
\tilde{\psi}_{\alpha} \\
\tilde{\psi}_{\beta}
\end{array}\right]=-k_{2}\left[\begin{array}{c}
\tilde{\psi}_{\alpha} \\
\tilde{\psi}_{\beta}
\end{array}\right]
$$

Hence, if only the parameter $k_{2}$ is a positive gain, the (24) ensures the errors converge to zero, and the convergence rate of error dynamic is determined by $k_{2}$. However, when $\omega_{e}$ is close to zero, the computed parameter may become ill-conditioned. To avoid this undesirable effect, we choose the observer poles as $k_{2}=\gamma\left\|\omega_{e}\right\|$; hence the observer gain is calculated as

$$
K=\left[\begin{array}{cc}
k_{1}+k^{\prime} & 0 \\
0 & k_{1}+k^{\prime} \\
-L_{d} \cdot\left(k_{1}+k^{\prime}\right) & -L_{d} \cdot \gamma \operatorname{sgn}\left(\omega_{e}\right) \cdot\left(k_{1}+k^{\prime}\right) \\
L_{d} \cdot \gamma \operatorname{sgn}\left(\omega_{e}\right) \cdot\left(k_{1}+k^{\prime}\right) & -L_{d} \cdot\left(k_{1}+k^{\prime}\right)
\end{array}\right]
$$

\subsection{Estimation of speed and position}

Conventionally, the rotor position can be estimated by using arc-angent function

$$
\hat{\theta}_{e}=\tan ^{-1}\left(\hat{\psi}_{\beta} / \hat{\psi}_{\alpha}\right)
$$

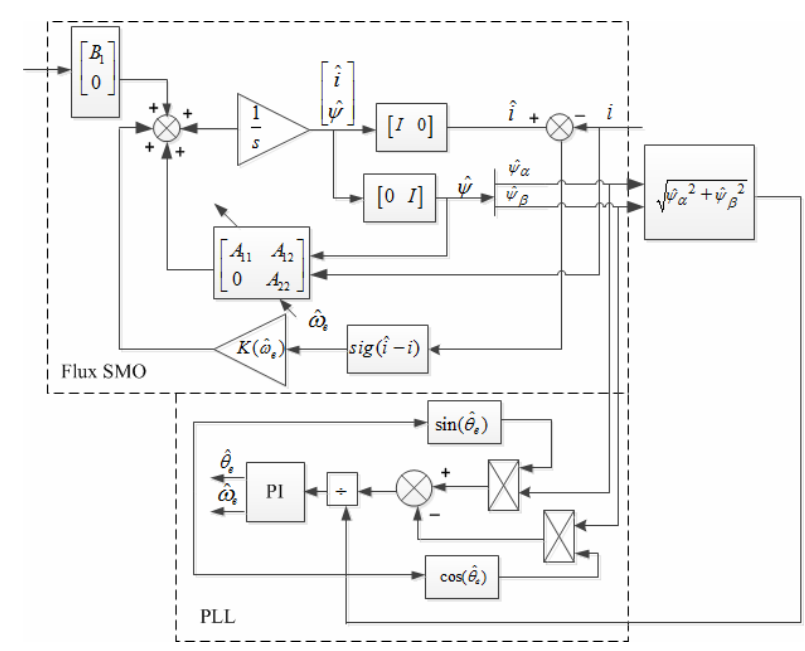

Fig. 1. Scheme of position estimation through PLL

However, the existence of noise and harmonics may influence the accuracy of the position estimation, especially at very low-speed, the obvious estimation error may occur using the arc-angent function. To improve the position estimation for mitigation of the adverse influence, a phaselocked loop (PLL) method is employed. This scheme can be comparatively represented as a simple linearized closedloop system shown in Fig. 1.

As shown in Fig. 1, after the normalization of the flux linkage, the equivalent position error signal can be expressed as

$$
\Delta \theta_{e}=\frac{1}{\sqrt{\hat{\psi}_{\alpha}^{2}+\hat{\psi}_{\beta}^{2}}}\left(-\hat{\psi}_{\alpha} \sin \left(\hat{\theta}_{e}\right)+\hat{\psi}_{\beta} \cos \left(\hat{\theta}_{e}\right)\right)
$$

The estimation of the electrical angular speed of the rotor is obtained using PI controller, i.e.,

$$
\left\{\begin{array}{l}
\dot{\hat{\omega}}_{e}=k_{i} \Delta \theta_{e} \\
\dot{\hat{\theta}}_{e}=\hat{\omega}_{e}+k_{p} \Delta \theta_{e}
\end{array}\right.
$$

where, the nonnegative gains $k_{p}$ and $k_{i}$ are selected as [15]

$$
k_{p}=2 \alpha, k_{i}=\alpha^{2}
$$

where, $a$ is the design parameter.

\section{Current Control of DT-IPMSM}

From the model of the DT-IPMSM, it seems that the two current loop control techniques of the three-phase motors can be easily extended to the six-phase drives, as depicted in Fig. 2. The phase currents are applied to the transformation matrix (1) to obtain the stator current 


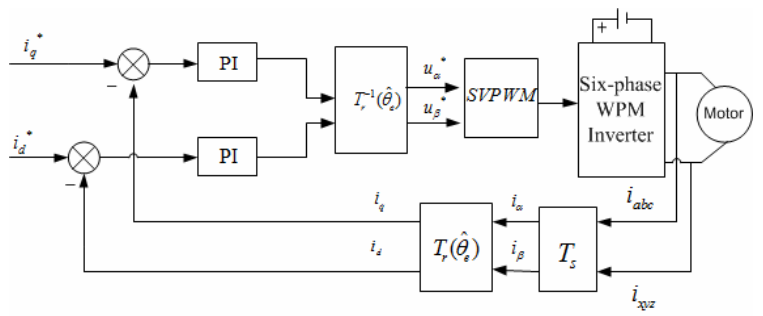

Fig. 2. The conventional two current loop controls for DTIPMSM

components in the stationary $(\alpha, \beta)$ reference frame. The $(d, q)$ current components in the synchronous reference frame are obtained by using a rotor position from the flux SMO. The outputs of the PI current regulators, after an inverse Park transformation, are the stator voltage reference components in stationary reference frame $(\alpha, \beta)$ to be applied to the SVPWM modulator.

The two current loop control strategy depicted in Fig. 2 is very simple, but it is not able to compensate for the inherent asymmetries of the drive. Due to the small asymmetries in the stator windings and supply voltages, the two sets of three-phase stator currents have rather different amplitudes depending on the operational conditions, and the harmonic currents in $\left(z_{1}, z_{2}\right)$ subsystem is not eliminated effectively. To obtain better controller performance, a current control technology with four current loop regulators in $(d, q)$ and $\left(z_{1}, z_{2}\right)$ subsystems are adopted in this paper. Moreover, in order to overcome the current coupling terms on $(d, q)$ subsystem, the decoupling and diagonal internal model control (DIMC) [8, 16] structure for current control in the drive. The command voltage are now given by

$$
\begin{aligned}
& u_{d}{ }^{*}=\beta\left(L_{d}+\frac{R}{s}\right)\left(i_{d}{ }^{*}-i_{d}\right)-\hat{\omega}_{e} L_{q} i_{q} \\
& u_{q}{ }^{*}=\beta\left(L_{q}+\frac{R}{s}\right)\left(i_{q}{ }^{*}-i_{q}\right)+\hat{\omega}_{e}\left(L_{d} i_{d}+\psi_{f}\right) \\
& u_{z 1}{ }^{*}=\beta\left(L_{z}+\frac{R}{s}\right)\left(i_{z 1}{ }^{*}-i_{z 1}\right) \\
& u_{z 2}{ }^{*}=\beta\left(L_{z}+\frac{R}{s}\right)\left(i_{z 2}{ }^{*}-i_{z 2}\right) .
\end{aligned}
$$

where, $\beta$ is the desired closed-up bandwidth as determined by the specified rise time of the current controller. The DIMC involves only a single parameter, so tuning of the controller to give specified performance is easier. The speed controller outputs the $q$-axis current reference $i_{q}{ }^{*}$, and the $z_{1}$-axis and $z_{2}$-axis current references $i_{z 1}{ }^{*}$ and $i_{z 2}{ }^{*}$ are set to zero, so the overall block diagram of the DT-IPMSM control scheme can be shown in Fig. 3.

To obtain better results and implement simply, it is suggested in this paper the dual three-phase SVPWM technique as shown in Fig. 4 is used for modulation in this paper, the detailed discussion can be seen in [17]. It is has many advantageous, such as existing algorithms and tested three-phase modulation methods can be effectively utilized,

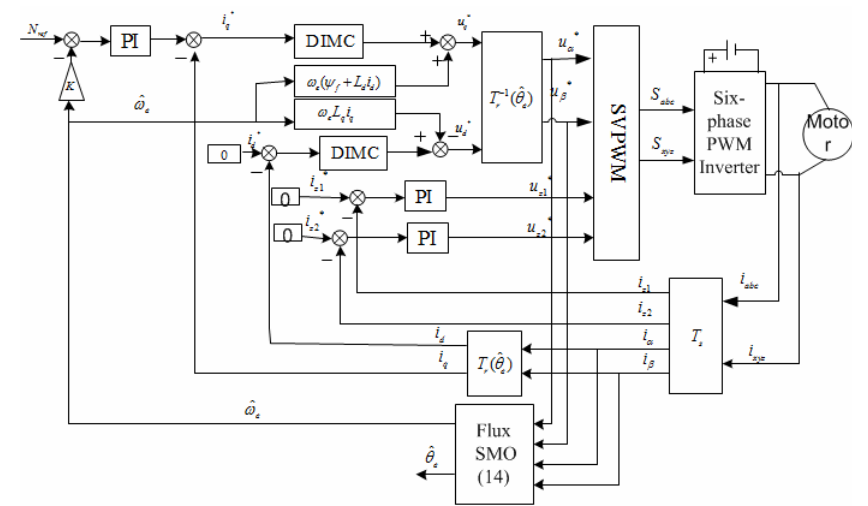

Fig.3 Block diagram of the sensorless flux SMO control drive scheme

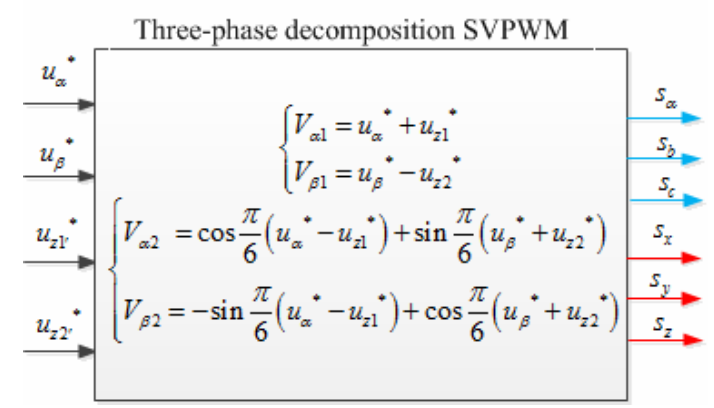

Fig. 4. The dual three-phase space vector classification PWM technique

which can save time and trouble. It also makes the method computationally efficient since years of extensive study and wide usage have made space vector modulation a very simple task.

\section{Simulation and Experiment Results}

To check the feasibility of the proposed rotor position and speed estimation schemes, the simulation and experimental studies are carried out with a reference to a 2 MW DT-IPMSM drives system. The block diagram of the sensorless flux observer control drives is presented in Fig. 5 , and the machine parameters are given in Table 1 . The same controller parameters are used both in simulation and experimental results. A stator current controller bandwidth $\beta$ of $30 \mathrm{rad} / \mathrm{s}$, and the PLL system bandwidth $a$ of $3 \mathrm{rad} / \mathrm{s}$

Table 1. the parameters of DT-IPMSM

\begin{tabular}{c|c}
\hline rate power & $2180 \mathrm{~kW}$ \\
\hline rate voltage & $690 \mathrm{~V}$ \\
\hline rate speed & $17 \mathrm{r} / \mathrm{min}$ \\
\hline rate frequency & $8.5 \mathrm{~Hz}$ \\
\hline rotor inertia & $16000 \mathrm{~kg} \cdot \mathrm{m}^{2}$ \\
\hline number of pole pairs & 30 \\
\hline stator resistance per phase $(R)$ & $0.0192 \Omega$ \\
\hline d-axis inductance $\left(L_{d}\right)$ & $4 \mathrm{mH}$ \\
\hline q-axis inductance $\left(L_{q}\right)$ & $5 \mathrm{mH}$ \\
\hline Permanent magnet flux $\left(\psi_{f}\right)$ & $10.228 \mathrm{~Wb}$ \\
\hline
\end{tabular}


are chosen. The parameters of flux observer are chosen as followings: $k_{1}+k^{\prime}=300, L_{d} \cdot \gamma=50$.

\subsection{Simulation results}

Figs. 5 and 6 show the two sets of simulation waveforms
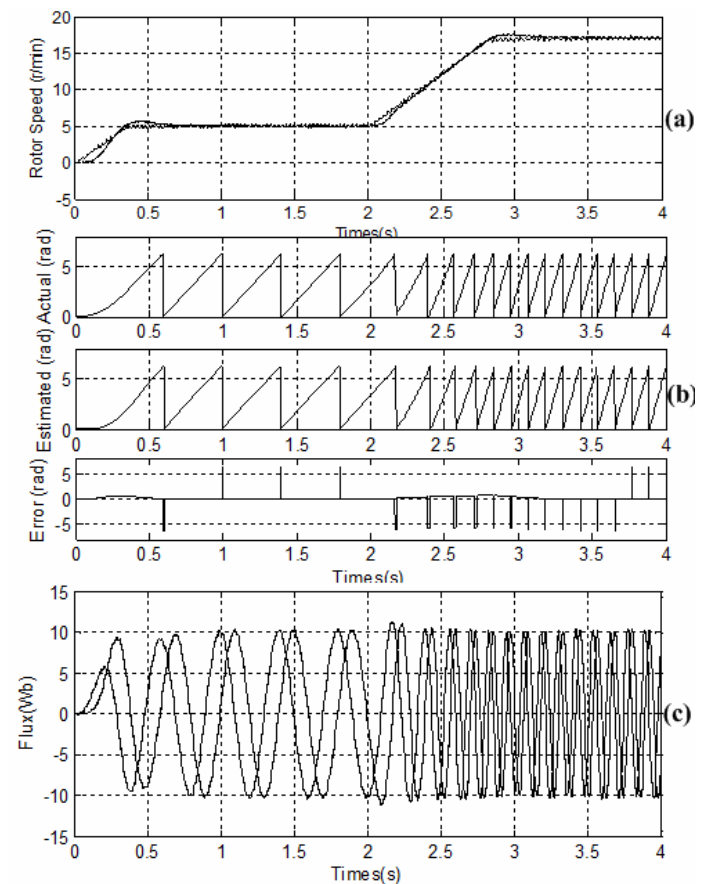

Fig. 5. The simulation waveforms obtained by the conventional SMO method using sign function: (a) Actual and estimated speeds; (b) Actual rotor position, estimated rotor position, and estimated error; (c) Estimated flux $\psi_{\alpha, \beta}$.
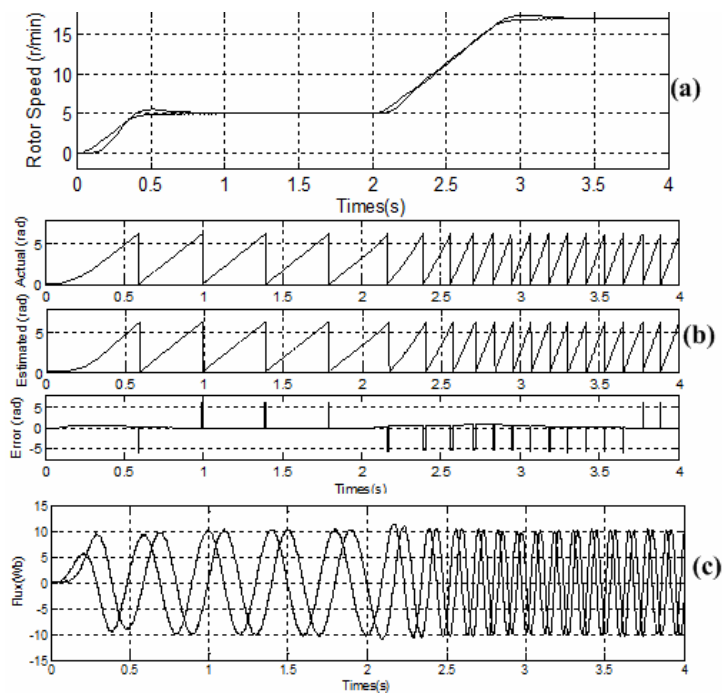

Fig. 6 The Simulation waveforms obtained by the conventional method using sign function: (a) Actual and estimated speeds; (b) Actual rotor position, estimated rotor position, and estimated error; (c) Estimated flux $\psi_{\alpha, \beta}$. when the reference speed is a step signal. In the simulation, the reference speed is changed from 5 to $17 \mathrm{r} / \mathrm{min}$, and the load torque is $5.6 \times 10^{5}$ N.m.

Fig. 5 displays the simulation waveform obtained by the conventional SMO method using a sign function. Fig. 6 shows the simulation waveform obtained by the method proposed in this paper. It can be seen from Figs. 5 that the sign function can cause to chattering phenomenon, the low pass filter and phase compensation part must be used, and therefore, the rotor position estimation accuracy is not high. However, it can be seen from Fig. 6 that the chattering phenomenon of the estimated rotor position and speed is reduced, and the accuracy of rotor position estimation is improved to some extent.

Fig. 7 shows the estimation performance of the proposed method when the parameters of DT-IPMSM vary. In the simulation, the reference speed is $17 \mathrm{r} / \mathrm{min}$, and the load torque is $5.6 \times 10^{5} \mathrm{~N}$ m. It can be seen from Fig. 7 that, when the resistance or the inductance of the motor changes, the estimated speed can still converge to the actual value, which verifies the robustness of the proposed approach.

\subsection{Experiment results}

The effectiveness of the proposed sensorless control scheme for $2 \mathrm{MW}-$ level DT-IPMSM drive is tested using the experiment setup shown in Fig. 8. In the experiment setup, a high-power back-to-back converter system is used to feed the drive system, and the controller and machine parameters are same with the simulation, and the sampling period of the control system is set as $50 \mu \mathrm{s}$, the dead-time is set as $10 \mu \mathrm{s}$, the switch frequency is set as $1 \mathrm{kHz}$. The overall system control algorithm is developed in Matlab/ Simulink, followed by implementation on an OPAL RTLab (Real-time Digital Simulator) controller board. The motor parameters are given in Table 1 .

Figs. 9 and 10 show the control performance when the DT-IPMSM is running with the reference speed steps up from 2 to 10 r/min. Fig. 9 shows the waveforms when the
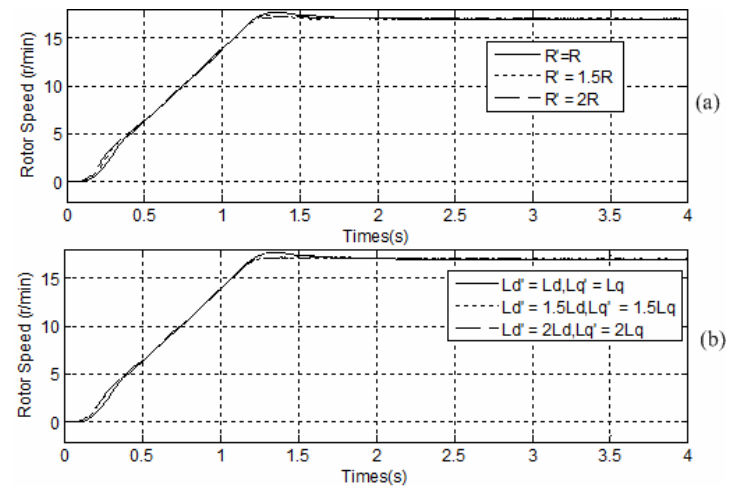

Fig. 7. Simulation waveforms when the parameters of DTIPMSM are changed: (a) Waveforms when the resistance is changed; (b) Waveforms when the inductance is changed. 


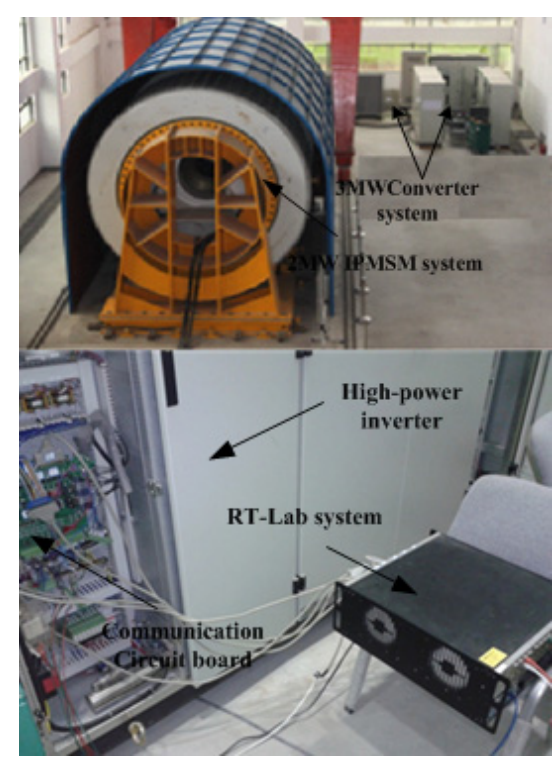

Fig. 8. The overall experiment setup
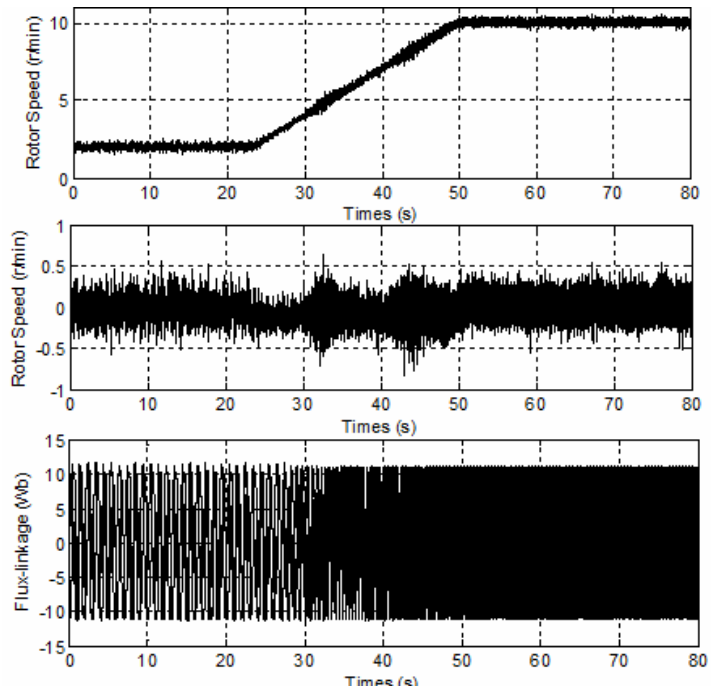

(a)
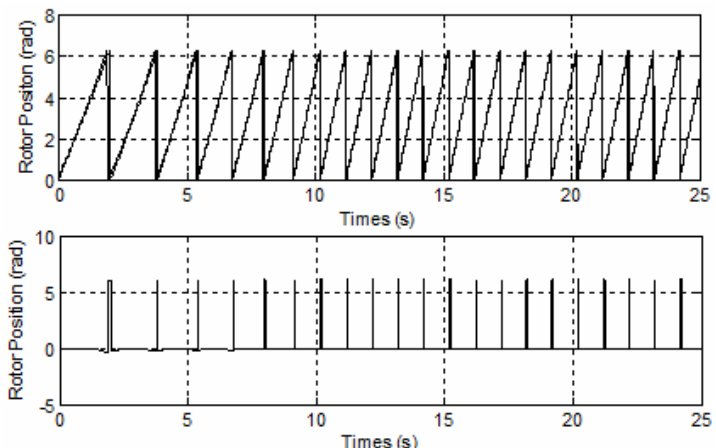

(b)

Fig. 9. Operating waveforms obtained by the conventional control method using a sign function: (a) From top to bottom are the estimated rotor speed, rotor error and estimated flux-linkage, respectively; (b) From top to bottom are the estimated rotor position, and estimated error, respectively.

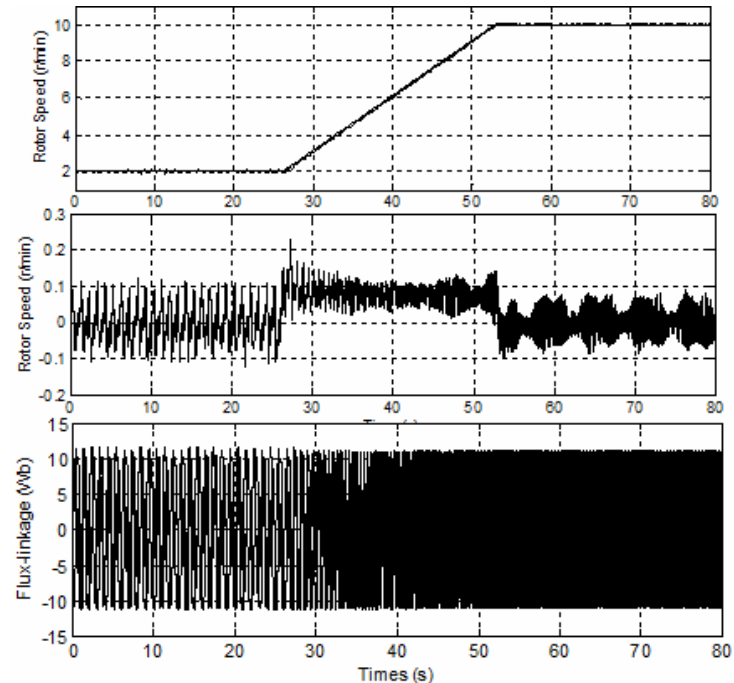

(a)
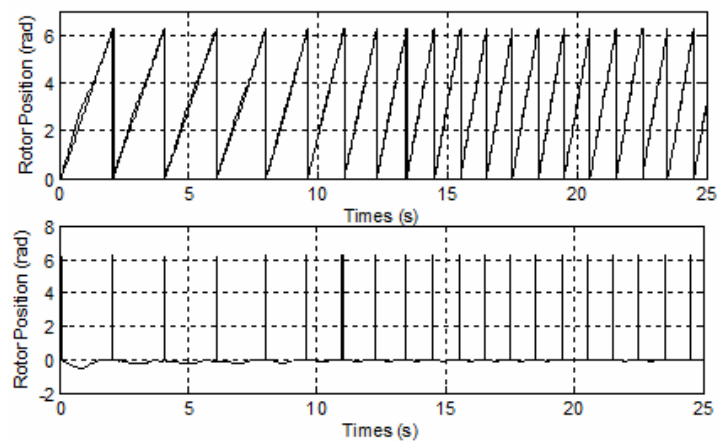

(b)

Fig. 10. Operating waveforms obtained by the proposed method using a sigmoid function: (a) From top to bottom are the estimated rotor speed, rotor error and estimated flux-linkage, respectively; (b) From top to bottom are the estimated rotor position, and estimated error, respectively.

conventional method based on the sign function and lowpass filter is adopted. As can be seen from Fig. 9, due to the use of the sign function, the chattering of the estimated rotor position and speed obtained by the traditional method is significant.

When the proposed flux SMO is employed, Fig. 10 presents a good dynamic performance of estimated speed, the estimated speed follows the reference speed very well, and the speed estimated error is very small. Especially, there is a small ripple $( \pm 0.1 \mathrm{r} / \mathrm{min})$ when DT-IPMSM drive runs in a stable speed range. And the chattering is reduced when the sign function is replaced by the sigmoid function, and the waveforms of the estimated rotor position and speed obtained by the proposed flux SMO are smooth.

\section{Conclusion}

In this paper, a novel flux linkage sliding-mode observer for DT-IPMSM sensorless drives has been proposed. To 
simply the machine model, an equivalent flux linkage concept is employed. The sign function is replaced by the sigmoid function to reduce the chattering, and the conventional SMO is improved. From the design process we can see that the presented observer has a simple structure with less control parameters. Meanwhile, the details of the observer parameters and the rotor position and speed estimators are given. The feasibility of the proposed scheme is verified and confirmed through simulation and extensive experiments.

\section{Acknowledgements}

The work described in this paper was fully supported by a grant from the Major State Basic Research Development Program of China (No.2013CB035601), and the Project supported by the Program for New Century Excellent Talents in University of Ministry of Education of China (No.NCET-11-0871).

\section{References}

[1] E. Levi, "Multiphase electric machines for variablespeed applications," IEEE Trans. Ind. Electron., vol. 55, pp. 1893-1908, May. 2008.

[2] E. Levi, R. Bojoi, F. Profumo, H. A. Toliyat, and S. Williamson," Multi- phase induction motor drives - a technology status review," IET Electr. Power Appl., vol. 1, pp.489-516, July. 2007.

[3] R. Bojoi, M. lazzari, F. Profumo, and A. Tenconi, "Digital field-oriented control for dual three-phase induction motor drives," IEEE Trans. Ind. Appl. vol. 39, pp. 752-759, May/June. 2003.

[4] G. K. Singh, K. Nam, and S. K. Lim, "A simple indirect field-oriented control scheme for multiphase induction machine," IEEE Trans. Ind. Electr. vol. 52, pp. 1177-1184, August. 2005.

[5] R. Wu, and G. Slemon, "A permanent magnet motor drive without a shaft sensor," IEEE Trans. Ind. Appl., vol. 27, pp. 1005-1011, May. 1991.

[6] S. Shinnaka. "New sensorless vector control using minimum-order flux state observer in a stationary reference frame for permanent-magnet synchronous motors," IEEE Trans. Ind. Electr., vol. 53, pp. 388398, April. 2006.

[7] Su Jianyong, Li Tiecai, Yang Guijie. PMSM sensorless control based on four-order hybrid sliding mode observer [J]. Proceedings of the CSEE, vol. 24, pp. 98-103, 2009.

[8] Chan T F, Wang W, Borsje P, etc. Sensorless permanent magnet synchronous motor drives using a reduced-order rotor flux observer [J]. IET Electric Power Applications, vol. 2, pp. 88-98, 2007.

[9] Z. Xu, M. F. Rahman, "An adaptive sliding stator flux observer for a direct-torque-controlled IPM synchronous motor drive" IEEE Trans. Ind. Electr. vol. 54, pp. 2398-2406, Oct. 2007.

[10] L. Yuan, F. Xiao. J Q Shen,etc, "Sensorless control of high- power interior permanent magnet synchronous motor drives at very low speed," IET Electrical power \& Applications, vol. 7, pp. 199-206, April. 2013.

[11] Yuan Lei, Shen Jian-qing, Xiao Fei, etc. Nonsingular terminal sliding-mode observer design for interior permanent magnet synchronous motor drive at very low-speed [J]. Acta Physiac Sinica, vol. 7, pp. 030501, 2013.

[12] Z. Chen, M. Tomita, S. Doki, and S. Okuma, "The pole assignment of adaptive sliding observers for BLM's sensorless control," in Nat. Conv. Rec. IEEJ, vol. 4, pp. 286-287, Mar. 1998.

[13] G. Foo ,and M. F. Rahman, “ sensorless sliding-mode MTPA control of an IPMSM synchronous motor drive using a sliding-mode observer and HF signal injection," IEEE Trans. Ind. Electron., vol. 57, pp. 1270-1278, April. 2010.

[14] Y. Zhao, and T. A. Lipo, "Space vector PWM control of dual three-phase induction machine using vector space decomposition," IEEE Trans. Ind. Appl., vol. 31, pp. 1100-1109, Sep./Oct. 1995.

[15] L. Harnefors and H. P. Nee, "A general algorithm for speed and position estimation of AC motors," IEEE Trans. Ind. Electr., vol. 47, pp. 77-83, February. 2000.

[16] L. Harnefors, and H. P. Nee, "Model-based current control of AC machines using the internal model control method," IEEE Trans. Ind. Appl., vol. 34, pp. 133-141, Jar./ Feb. 1998.

[17] R. Bojoi, A. Tenconi, F. Profumo, G. Griva, and D. Martinello, "Complete analysis and comparative study of digital modulation techniques for dual three-phase AC motor drives," in Proc. IEEE PESC'02, pp. 851857, 2002.

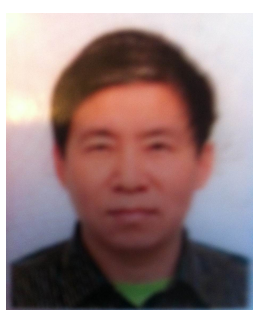

Jian-Qing Shen received the Ph.D. degree from Naval University of Engineering (NUE) in 2007, and is presently a research fellow at NUE. His areas of research are control method design of new type of electrical machine, power electronics and its applications in industry and power system, and power system integration technique applications

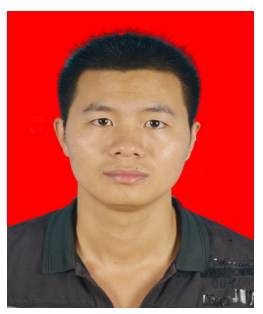

Lei Yuan received his B.S degree from Naval University of Engineering (NUE) in 2010, and is currently working toward the Ph.D. degree at NUE. His current interests include power electronics and the control method design of new type of electrical machine. 


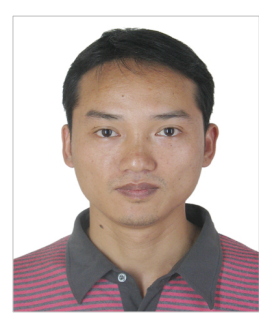

power system.
Ming-Liang Chen received the Ph.D. degree from Naval University of Engineering (NUE) in 2008, and is presently an associate research fellow at NUE. His areas of research are control method design of new type of electrical machine, power electronics and its applications in industry and

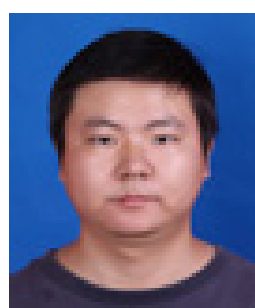

Zhen Xie received the B.S and Ph.D. degrees from Naval University of Engineering (NUE) in 2009 and 2013. His current interests include highpower power electronics technique applications. 